\title{
Asymptomatic epiploic appendage with torsion in laparoscopic surgery: a case report and literature review
}

\author{
Peiming Sun ${ }^{1} \wedge$, Jianwu Yang ${ }^{1} \wedge$, Hongchang Ren $^{1}$, Xiaobo Zhao ${ }^{2}$, Hongwei Sun ${ }^{1} \wedge$, Jie Cui ${ }^{1}$, \\ Heming Yang $^{1} \wedge$, Yan $\mathrm{Cui}^{{ }^{\wedge} \wedge}$ \\ ${ }^{1}$ Department of General Surgery, Strategic Support Force Medical Center, Beijing, China; ${ }^{2}$ Department of Pathology, Strategic Support Force \\ Medical Center, Beijing, China \\ Correspondence to: Hongwei Sun, MD, PhD; Heming Yang, MD, PhD. Department of General Surgery, Strategic Support Force Medical Center, 9 \\ Anxiangbeili, Chaoyang District, Beijing 100101, China. Email: shwsport@yeah.net; yhming306@163.com.
}

\begin{abstract}
Torsion of an epiploic appendage may result in epiploic appendagitis, which is a rare cause of acute abdominal pain. However, no previous reports have described an asymptomatic twisted epiploic appendage found during laparoscopic surgery to the best of our knowledge. This case describes a 66-year-old man who was admitted to our medical center with yellowness of the skin and eyes that had lasted over two months. Physical examination showed slight yellow staining of the skin and sclera. Blood analysis indicated liver dysfunction and jaundice. Routine blood, C-reactive protein (CRP), and levels of tumor markers were normal. The contrast-enhanced abdominal and pelvic computed tomography, magnetic resonance imaging, and magnetic resonance cholangiopancreatography revealed gallbladder atrophy and choledocholithiasis. The patient underwent laparoscopic surgery for the removal of the choledocholithiasis. The laparoscopic exploration unexpectedly revealed a twisted and ischemic epiploic appendage, which was surgically removed. The postoperative pathological examination uncovered necrosis of adipocytes and vascular obstruction, but there was no inflammation of the epiploic appendage. The patient had a satisfactory recovery during the 16-month follow-up period. This case describes and provides a feasible management approach for an asymptomatic twisted epiploic appendage discovered during surgery and highlights its pathological characteristics, reflecting the early stage of epiploic appendagitis.
\end{abstract}

Keywordsa Epiploic appendage; torsion; asymptomatic; laparoscopic surgery; case report

Submitted Oct 30, 2020. Accepted for publication Feb 24, 2021.

doi: $10.21037 /$ apm-20-2128

View this article at: http://dx.doi.org/10.21037/apm-20-2128

\section{Introduction}

Torsion of an epiploic appendage is a major cause of primary epiploic appendagitis $(1,2)$. Epiploic appendagitis can cause acute abdominal pain but may be misdiagnosed due to its very low incidence and the lack of clear clinical signs $(3,4)$. Although previous literature has focused on primary epiploic appendagitis caused by the torsion of an epiploic appendage, an asymptomatic twisted epiploic appendage found during laparoscopic surgery has not been reported previously. In this paper, we report a case of an asymptomatic epiploic appendage with torsion, which was unexpectedly discovered during laparoscopic surgery. The twisted epiploic appendage was surgically removed to prevent associated symptoms and complications. We present the following case following the CARE reporting checklist (available at http://dx.doi. org/10.21037/apm-20-2128).

^ ORCID: Peiming Sun, 0000-0003-1162-5412; Jianwu Yang, 0000-0002-4021-923X; Hongwei Sun, 0000-0003-4156-8340; Heming Yang, 0000-0002-2867-6809; Yan Cui, 0000-0002-6833-5611. 

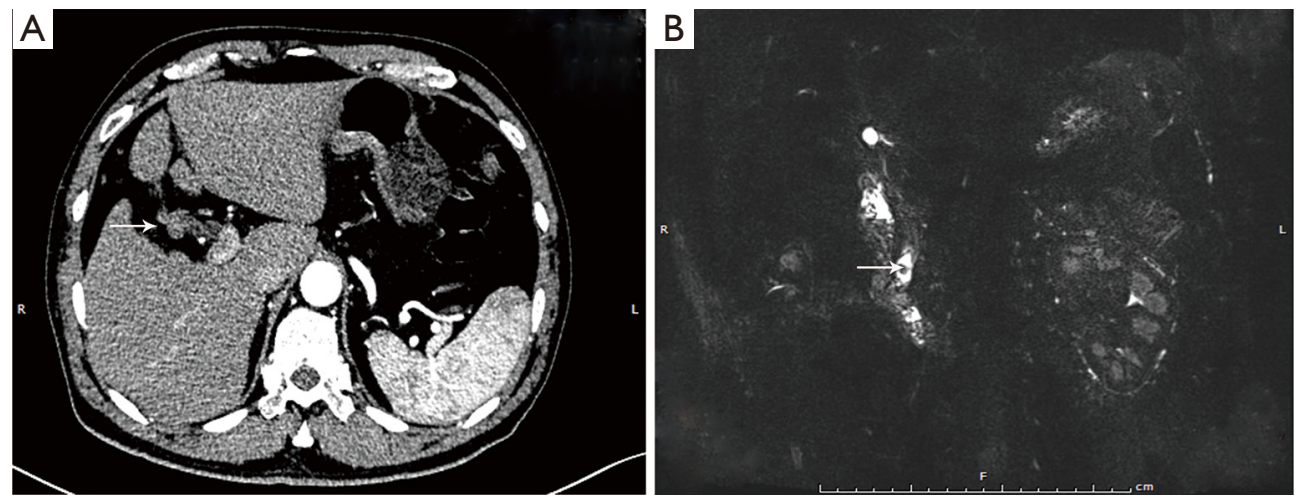

Figure 1 Preoperative imaging examinations. The imaging examinations showed gallbladder atrophy and choledocholithiasis. The contrast enhanced abdominal and pelvic CT shows that the gallbladder volume is reduced, and the structure of the gallbladder is unclear (arrow, A). The magnetic resonance imaging and magnetic resonance cholangiopancreatography reveal the stone incarceration on the distal common bile duct (arrow, B).

\section{Case presentation}

A 66-year-old male presented to the Department of General Surgery of Strategic Support Force Medical Center on March $16^{\text {th }}, 2019$, with yellowness of the skin and eyes; these symptoms were present for over two months. The patient had first observed slight yellow staining of the skin and eyes in December 2018. Since March $6^{\text {th }}, 2019$, and with the aggravation of the skin and eyes' yellowness, he felt abdominal pain in the right upper quadrant, had a fever of $38.7^{\circ} \mathrm{C}$, experienced shivering, and his urine was the color of dense tea. He did not experience abdominal distention, vomiting, or radiative, or referred pain. He received antimicrobial treatment and hepatoprotective therapies for five days in the local hospital following diagnoses of choledocholithiasis, acute cholangitis, and liver dysfunction. This resulted in the disappearance of the abdominal pain and the yellowness of the skin and eyes, and his body temperature returned to normal. He had a ten-year history of gallstones and, in December 2017, had undergone a cholecystostomy to remove stones due to acute cholecystitis and gallbladder gangrene. He had no other medical history.

Physical examination revealed slight jaundice of the skin and sclera. There was no abdominal pain and tenderness, and the Murphy sign was negative. He had lost approximately $2 \mathrm{~kg}$ of body weight over the past two weeks due to diet control, resulting in a body mass index (BMI) of 23.26. Blood biochemistry showed liver dysfunction and jaundice, including an alanine transaminase (ALT) level of $124 \mathrm{U} / \mathrm{L}$, an aspartate aminotransferase (AST) level of $77 \mathrm{U} / \mathrm{L}$, and a $\gamma$-glutamyltranspeptidase $(\gamma-\mathrm{GT})$ level of $139 \mathrm{U} / \mathrm{L}$. Total bilirubin was $86.9 \mu \mathrm{mol} / \mathrm{L}$, conjugated bilirubin was $44.5 \mu \mathrm{mol} / \mathrm{L}$, and unconjugated bilirubin was $42.4 \mu \mathrm{mol} / \mathrm{L}$. Routine blood, C-reactive protein (CRP), and levels of tumor markers were normal. The contrastenhanced abdominal and pelvic computed tomography (AP$\mathrm{CT}$ ), magnetic resonance imaging (MRI), and magnetic resonance cholangiopancreatography (MRCP) revealed gallbladder atrophy (Figure $1 A$ ) and stone incarceration on the distal common bile duct (Figure 1B). No other findings were reported upon image examination, resulting in the preoperative diagnosis of gallbladder atrophy and choledocholithiasis.

During laparoscopic surgery, a cholecystectomy, choledochotomy, and exploration of the bile duct and $\mathrm{T}$ tube drainage were performed to remove the stones. An ischemic epiploic appendage observed in the sigmoid colon's antimesenteric border was found accidentally during the laparoscopic exploration (Figure 2A). Further investigation revealed that the epiploic appendage was twisted on the root, and the adjacent colon wall looked healthy. The epiploic appendage pedicle was clipped with a titanium clip (Figure $2 B$ ) and cauterized using bipolar electrocautery. Subsequently, the epiploic appendage was excised and removed from the abdomen using a sterile Endo Bag. The tissue was curved and measured $3 \mathrm{~cm}$ in length (Figure $3 A$ ). After straightening, it measured $5.5 \mathrm{~cm}$ in length and had a diameter of $0.5-0.8 \mathrm{~cm}$. The histopathological examinations also revealed intravascular thrombosis, thrombus organization, and blockage of the adipose tissue's vascular cavity. The peripheral capillaries were hyperemic and expansive (Figure 3B). The T-tube 

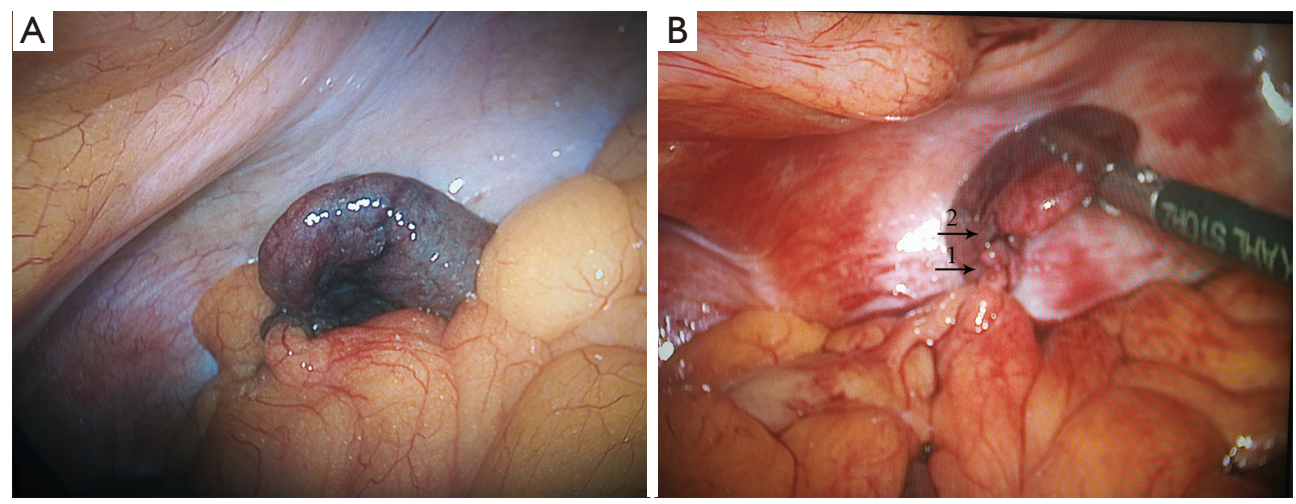

Figure 2 Surgical findings. The laparoscopic exploration revealed a twisted and ischemic epiploic appendage and then was surgically removed. The intraoperative photograph shows the ischemic and twisted epiploic appendage in the sigmoid colon's antimesenteric border $(\mathrm{A}, \mathrm{B})$. The epiploic appendage is twisted at the root (arrows 1, B), and the adjacent colon wall is normal (B). The epiploic appendage pedicle is clipped with a titanium clip (arrows 2, B) and surgically removed.
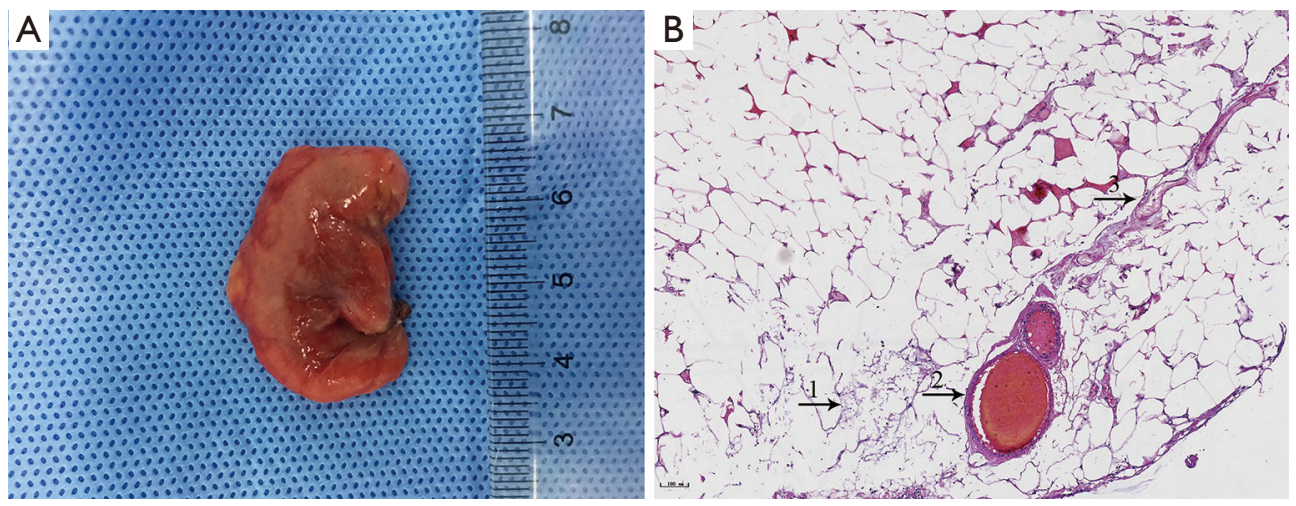

Figure 3 Postoperative histopathological findings. The pathological examination uncovered necrosis of adipocytes and vascular obstruction, but there was no inflammation of the epiploic appendage. The tissue is curved and measures $3 \mathrm{~cm}$ in length (A). The pathologic diagnosis (H\&E stain, $\times 100$ ) shows necrotic adipocytes as ghost-like cells (arrows 1, B), intravascular thrombosis (arrows 2, B), hyperemia, and expansive peripheral capillaries (arrows $3, \mathrm{~B}$ ). There are no infiltrating inflammatory cells.

cholangiography showed patency of the common bile duct on postoperative day 13 (Figure 4). The patient was discharged without complications, the T-tube was removed four weeks after surgery, and the patient had a satisfactory recovery during the 16 months of follow-up.

All procedures performed in studies involving human participants were in accordance with the ethical standards of the institutional and/or national research committee(s) and with the Helsinki Declaration (as revised in 2013). Written informed consent was obtained from the patient for publication of this case report and any accompanying images.

\section{Discussion}

Epiploic appendagitis is an inflammation of the epiploic appendage and a rare cause of acute abdominal pain that often manifests with acute onset of pain in the left or right lower quadrant. Its symptoms can mimic and be mistaken for many other abdominal diseases, including acute diverticulitis or appendicitis. As reported in previous literature, torsion of epiploic appendages usually causes epiploic appendagitis. This report described a very rare case of an asymptomatic epiploic appendage with torsion, which was found during laparoscopic surgery. Here we provide a 


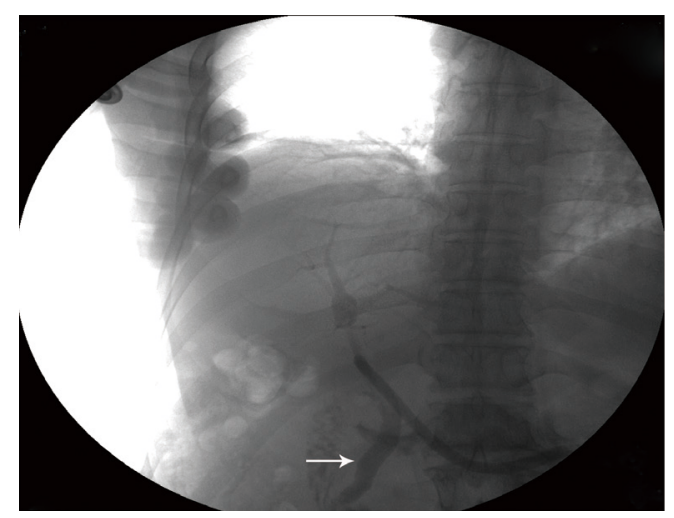

Figure 4 Postoperative imaging examinations. The imaging examination showed the common bile duct was unobstructed. The T-tube cholangiography shows patency of the common bile duct after surgery (arrow).

summary of epiploic appendagitis and indicate the clinical significance of this case.

\section{The physiology and function of epiploic appendages}

Epiploic appendages are small fat pouches that are pedunculated, adipose outpouchings abutting the colon's serosal surface $(1,5)$. Approximately 50-100 epiploic appendages are extending from the cecum to the rectosigmoid junction in adults. Epiploic appendages appear more abundantly in the rectosigmoid junction and transverse colon but can also be found at the appendix's base while being absent in the rectum $(1,6)$. Epiploic appendages are usually $1-2 \mathrm{~cm}$ in thickness and $0.5-5 \mathrm{~cm}$ long but can sometimes be $10-15 \mathrm{~cm}$ long in patients who are obese or have lost weight within a short period $(4,7)$. The vascular supply of epiploic appendages depends on one or two small feeding arteries originating from the colon's margin branch artery, while tortuous veins pass through the narrow pedicle to ensure venous drainage $(6,7)$. This is the main reason for the increased risk of torsion with subsequent ischemia or hemorrhage of the epiploic appendage. An epiploic appendage function is unclear but may include serving as a protective fat pad of the colon, fat storage, absorption of moisture and electrolytes, and intestinal immunity $(4,6-8)$.

\section{The clinical features of epiploic appendagitis}

Since the first report by Dockerty et al. in 1956 (9), epiploic appendagitis has been considered a rare cause of abdominal pain, with an incidence of only 8.8 cases per million people every year. It can occur at any age and is more common between the second and fifth decades of life, and it more frequently affects men than women $(4: 1)(7,8)$. Epiploic appendagitis can occur in any segment of the colon but is most frequently found in the sigmoid colon (48\%), followed by the descending colon (28\%), ascending colon (17\%), and transverse colon (7\%) (10). Torsion of epiploic appendages compromising the blood supply is usually the main cause of primary epiploic appendagitis, but its draining vein's thrombosis can also cause it. Secondary epiploic appendagitis usually results from adjacent inflammation, such as appendicitis and diverticulitis $(4,11)$. It has been reported that obesity, strenuous exercise, rapid weight loss, colonic diverticula, and hernias are potential risk factors for the development of epiploic appendagitis $(7,8,11)$.

Epiploic appendagitis does not exhibit unique clinical symptoms, and the most important sign is acute or subacute localized non-radiating pain in the left or right lower quadrant $(1,4,5,8)$. Some studies have indicated that left lower quadrant pain is the most common presentation (69-89\%), followed by right lower quadrant pain (8-16\%), and pain in the right and left upper quadrant and other locations (1.5-3\%) (6). The symptoms could mimic and be mistaken for colonic diverticulitis, acute appendicitis, or even acute cholecystitis or a gynecological disorder $(3,4,6,10)$. There are no other relevant symptoms in the majority of patients. Sometimes the patient may have nausea and vomiting; however, fever and diarrhea are unusual $(1,2,8,11)$. The physical examination usually reveals focal non-migratory abdominal pain associated with rebound tenderness without rigidity in the lower abdominal quadrants. The pain is typically localized and is not aggravated with physical movement $(4,5,7)$. Therefore, the patient usually can present the location of the pain accurately when admitted to the hospital. Some researchers have also reported that the pain could be aggravated by coughing and abdominal stretching (12). Laboratory tests, including white blood cell count, erythrocyte sedimentation rate, and CRP levels, are usually within the normal range, while the white blood cell count may occasionally show a mild increase due to an inflammatory response $(7,11,12)$.

\section{The diagnosis of epiploic appendagitis}

Diagnosis of epiploic appendagitis is difficult because of its variable presentation and unspecific symptoms. Cases 
are often found while excluding the common causes of acute abdominal pain, sometimes during exploratory surgery (12). With the development of imaging examinations, various ultrasound (US), CT, and MRI findings have been described, which help provide a proper preoperative diagnosis. A CT scan is the first preoperative diagnostic approach for epiploic appendagitis. Normal epiploic appendages cannot be distinguished from other adipose structures on a CT scan unless surrounded by intraperitoneal fluid or inflammation. The typical CT image includes: (I) a solitary fat-dense ovoid lesion abutting the colon wall anteriorly, measuring $1.5-5 \mathrm{~cm}$ in axial diameter; (II) inflammatory changes surrounding the ovoid lesion, showing a peripheral high attenuation rim (2-3 mm thick); (III) a central high-intensity dot which indicates central venous pedicle thrombosis within the epiploic appendage $(4,5,8)$. The "hyperattenuating ring sign" is reported as a notable radiological feature and serves as the primary criterion for diagnosing epiploic appendagitis. The adjacent colonic wall is usually healthy or with slight inflammation, which appears in imaging as a characteristic "fat stranding" sign $(5,8)$. The other major value of a CT scan is to exclude the common causes of acute abdominal pain, such as diverticulitis or appendicitis. Characteristic US findings show an oval, non-compressible, hyperechoic, localized mass directly under the site of maximum pain, which is adjacent to the anterior peritoneal wall and is fixed during breathing. The mass frequently presents with a subtle hypoechoic rim, representing the inflamed peritoneum. Furthermore, no central blood flow is visible on color Doppler due to torsion or thrombosis $(2,13,14)$. MRI is rarely used to diagnose epiploic appendagitis, and T1- and T2-weighted images will present a focal lesion shown as an ovoid fat intensity with a central dot and surrounding enhanced rim $(15,16)$. Adhesions, abscess formation, peritonitis, bowel obstruction, intussusception, and intraperitoneal loose bodies are the general complications of epiploic appendagitis $(1,4,11)$.

\section{The treatment of epiploic appendagitis}

The majority of studies have reported that epiploic appendagitis is a self-limiting disease, and most cases were successfully treated with nonsteroidal anti-inflammatory drugs (NSAIDS) within an average of 10 days. However, this treatment is associated with a high rate of recurrence $(17,18)$. The CT findings can return to normal for about six months (8). Antibiotic therapy is rarely required unless complications such as an abscess are present $(4,11)$. Surgery is performed to remove the affected appendage when patients do not respond to conservative medical therapy or recurrences or complications such as abscess formation, obstruction, and intussusception $(2,7,8)$. Laparoscopic surgery is an excellent option for treating acute abdominal pain of unclear etiology, including epiploic appendagitis. The surgeon can thoroughly explore the abdominal cavity, diagnose, and remove the lesions simultaneously, all with minimal invasiveness $(12,19,20)$.

\section{The pathological characteristics of epiploic appendagitis}

Epiploic appendagitis is thought to be an inflammatory disease that presents with ischemia and infarction with aseptic fat necrosis and spontaneous venous thrombosis $(1,4,7,14)$. As epiploic appendagitis is considered a nonsurgical disease and radiologic examinations are in development, there have been few publications in recent years focusing on this pathology. Some reports revealed that this pathology's characteristics are acute inflammation and fat necrosis of the epiploic appendage $(12,19,20)$.

\section{The clinical significance of this case}

In our case, as the patient had not had any recent strenuous exercise and had a healthy BMI, the cause of the epiploic appendage torsion was possibly the rapid weight loss due to diet control. There was no acute abdominal pain during the present history, and the twisted epiploic appendage did not show in the preoperative imaging examinations but was found accidentally during laparoscopic surgery. This may be because the torsion was at an early stage, even though there was necrosis of adipocytes and intravascular thrombosis. However, postoperative pathological examinations showed that the epiploic appendage was not inflamed. This may be the characteristic feature of our case that has not been seen in previous reports. On the other hand, the symptoms caused by the twisted epiploic appendage may have been covered by the pain caused by gallbladder atrophy and choledocholithiasis. Our case also indicates that a complete exploration of the abdominal cavity during laparoscopic surgery is necessary and essential for detecting an asymptomatic twisted epiploic appendage and many other unexpected diseases. The twisted epiploic appendage should be surgically removed to prevent possible complications, 
even when there are no clinical symptoms.

In conclusion, our case report describes and provides a feasible management approach for an asymptomatic twisted epiploic appendage discovered during surgery. Furthermore, this case highlights the advantages of laparoscopic surgery. Notably, our case provides a twisted epiploic appendage's pathological characteristics, which may be the early stage of epiploic appendagitis.

\section{Acknowledgments}

We thank our coworkers of the departments of radiology, laboratory, electrocardiogram, and operating room for their invaluable technical help. We also thank AME Editing Service for their professional help to improve the English language of the paper.

Funding: None.

\section{Footnote}

Reporting Checklist: The authors have completed the CARE reporting checklist. Available at http://dx.doi.org/10.21037/ apm-20-2128

Conflicts of Interest: All authors have completed the ICMJE uniform disclosure form (available at http://dx.doi. org/10.21037/apm-20-2128). The authors have no conflicts of interest to declare.

Ethical Statement: The authors are accountable for all aspects of the work in ensuring that questions related to the accuracy or integrity of any part of the work are appropriately investigated and resolved. All procedures performed in studies involving human participants were in accordance with the ethical standards of the institutional and/or national research committee(s) and with the Helsinki Declaration (as revised in 2013). Written informed consent was obtained from the patient for publication of this case report and any accompanying images.

Open Access Statement: This is an Open Access article distributed in accordance with the Creative Commons Attribution-NonCommercial-NoDerivs 4.0 International License (CC BY-NC-ND 4.0), which permits the noncommercial replication and distribution of the article with the strict proviso that no changes or edits are made and the original work is properly cited (including links to both the formal publication through the relevant DOI and the license).
See: https://creativecommons.org/licenses/by-nc-nd/4.0/.

\section{References}

1. Gourgiotis S, Oikonomou C, Veloudis G, et al. The Diagnostic Dilemma of Primary Epiploic Appendagitis and How to Establish a Diagnosis. Oman Med J 2016;31:235-7.

2. Nadida D, Amal A, Ines M, et al. Acute epiploic appendagitis: Radiologic and clinical features of 12 patients. Int J Surg Case Rep 2016;28:219-22.

3. Quaas AM, Mueller PR, Kickham JM. Epiploic appendagitis mimicking pelvic inflammatory disease (PID). Eur J Obstet Gynecol Reprod Biol 2008;140:134-5.

4. Giannis D, Matenoglou E, Sidiropoulou MS, et al. Epiploic appendagitis: pathogenesis, clinical findings and imaging clues of a misdiagnosed mimicker. Ann Transl Med 2019;7:814.

5. Choi YU, Choi PW, Park YH, et al. Clinical characteristics of primary epiploic appendagitis. J Korean Soc Coloproctol 2011;27:114-21.

6. Suresh Kumar VC, Mani KK, Alwakkaa H, et al. Epiploic Appendagitis: An Often Misdiagnosed Cause of Acute Abdomen. Case Rep Gastroenterol 2019;13:364-8.

7. Sand M, Gelos M, Bechara FG, et al. Epiploic appendagitis--clinical characteristics of an uncommon surgical diagnosis. BMC Surg 2007;7:11.

8. Giambelluca D, Cannella R, Caruana G, et al. CT imaging findings of epiploic appendagitis: an unusual cause of abdominal pain. Insights Imaging 2019;10:26.

9. Dockerty MB, Lynn TE, Waugh JM. A clinicopathologic study of the epiploic appendages. Surg Gynecol Obstet 1956;103:423-33.

10. Patel NB, Wenzke DR. Evaluating the Patient with Right Lower Quadrant Pain. Radiol Clin North Am 2015;53:1159-70.

11. Schnedl WJ, Krause R, Tafeit E, et al. Insights into epiploic appendagitis. Nat Rev Gastroenterol Hepatol 2011;8:45-9.

12. Vázquez-Frias JA, Castañeda $P$, Valencia $S$, et al. Laparoscopic diagnosis and treatment of an acute epiploic appendagitis with torsion and necrosis causing an acute abdomen. JSLS 2000;4:247-50.

13. Matsunaga H, Fujii Y, Taniguchi N. Ultrasonographic findings in epiploic appendagitis. J Med Ultrason (2001) 2010;37:31-2.

14. Hasbahceci M, Erol C, Seker M. Epiploic appendagitis: is there need for surgery to confirm diagnosis in spite 
of clinical and radiological findings? World J Surg 2012;36:441-6.

15. Sirvanci M, Balci NC, Karaman K, et al. Primary epiploic appendagitis: MRI findings. Magn Reson Imaging 2002;20:137-9.

16. Lo Re G, Carcione P, Vernuccio F, et al. Primary epiploic appendagitis in a pediatric patient: prominent role of Magnetic Resonance Imaging in the diagnosis. Minerva Pediatr 2015;67:529-30.

17. Lorente C, Hearne CB, Taboada J. Recurrent epiploic appendagitis mimicking appendicitis and cholecystitis.

Cite this article as: Sun P, Yang J, Ren H, Zhao X, Sun H, Cui J, Yang H, Cui Y. Asymptomatic epiploic appendage with torsion in laparoscopic surgery: a case report and literature review. Ann Palliat Med 2021;10(7):8384-8390. doi: 10.21037/apm-20-2128
Proc (Bayl Univ Med Cent) 2017;30:44-6.

18. Michael A, Zakry Y, Hanif H. Epiploic Appendagitis: An uncommon surgical diagnosis. Med J Malaysia 2017;72:128-9.

19. Bunni J, Corrigan A, Jacob K, et al. Epiploic appendagitis: a case report highlighting correlation between clinical features, computed tomography images and laparoscopic findings. Int J Surg 2010;8:401-3.

20. Tabbara TA, Alassaf OY, Kaouas MC. Acute epiploic appendigitis: Diagnostic and laparoscopic approach. Int J Surg Case Rep 2018;44:157-60. 